\title{
Research Paper: Evaluation of Electromyographic Activity of Upper Trapezius Muscle Fatigue in Patients with Latent Myofascial Trigger Point: A Randomized Control Trial
}

\author{
Roya Mehdikhani $^{1}$ D, Gholam Reza Olyaei ${ }^{1 *}$ (D), Mohammad Reza Hadian', Saeed Talebian Moghadam¹, Azadeh Shadmehr ${ }^{1}$
}

1. Department of Physiotherapy, School of Rehabilitation, Tehran University of Medical Sciences, Tehran, Iran.

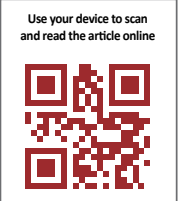

Citation: Mehdikhani R, Olyaei GR, Hadian MR, Talebian Moghadam S, Shadmehr A. Evaluation of Electromyographic Activity of Upper Trapezius Muscle Fatigue in Patients with Latent Myofascial Trigger Point: A Randomized Control Trial. Journal of Modern Rehabilitation. 2018; 12(4):209-216. http://dx.doi.org/10.32598/JMR.V12.N4.209

Article info:

Received: 03 May 2018

Accepted: 10 Aug 2018

Available Online: 01 Oct 2018

Keywords:

Trapezius muscle, Electromyography, Triggers points, Fatigue

\begin{abstract}
A B STRACT
Introduction: Latent Myofascial Trigger Points (LMTrPs) are defined as certain pain-free hyperirritable spots in a taut band of muscle, which lead to alternation in muscle activation pattern in both loaded and unloaded conditions. Fatigue can alter the stability of the cervical spine because of transferring loads to the passive connective tissues and also increasing muscle activity. Few studies have investigated the effects of fatigue on the parameters of the upper trapezius muscle as a more common muscle to MTrPs. This study attempts to examine the electromyographic activity of the upper trapezius muscle fatigue during shoulder elevation.
\end{abstract}

Materials and Methods: Thirty-six right-handed subjects without upper extremity disorders took part in this study. The highest measured force level was assumed to be the Maximal Voluntary Contraction (MVC) of the trapezius. A sustained submaximal contraction of the trapezius was performed. The subjects were asked to sustain a unilateral $(80 \%) \mathrm{MVC}$ isometric shoulder elevation until the force gauge monitor showed $(50 \%)$ of MVC in at least three minutes.

Results: The Root Mean Square (RMS norm) of the sustained trapezius contractions showed differences between the groups. The Myocardial Depressant Factor (MDF) parameters of the left and right sides of both healthy subjects and patients were significantly different $(\mathrm{P}<0.001)$.

Conclusion: The increase in RMS is related to the recruitment of additional motor units and also an increased firing rate. These are necessary to compensate for the loss of force. This accumulation also inhibits the excitability of the muscle membrane, thereby causing a decrease in the firing rate and, consequently, a decrease in Median Frequency (MF). 


\section{Introduction}

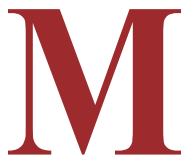

yofascial Trigger Points (MTrPs) are hyperirritable spots in the taut band of skeletal muscle which is the primary cause of discomfort and dysfunction in the upper limb.According to previous studies, Latent Myofascial Trigger Points (LMTrPs) are typically found in the scapular stabilizer muscles of painfree individuals; especially in the upper trapezius of the dominant side [1-3].

There is evidence showing relationship between highly repetitive work and neck/shoulder musculoskeletal disorders. Repetitive movements of sustained arm or hand at work place can affect the neck/shoulder musculature due to extra load. Fatigue can alter cervical spine stability because of the removal of load from the passive connective tissues and also due to increase in muscle activity. Trapezius muscle has three distinct parts. The upper trapezius part originates from the superior nuchal line to the ligamentum nuchae above C7 spinal process and has insertion in the posterior border of the distal third of the clavicle [4].

Usually, an increase in the amplitude of the ElectroMyography (EMG) signals together with a shift of the power spectrum towards low frequencies is followed by fatigue development at high level contractions [5-9]. Muscle activity can be studied by Surface EMG which is commonly used in biomechanics and ergonomics research $[4,10]$. Few studies have investigated the effects of fatigue on the parameters of the upper trapezius muscle as a more common muscle to MTrPs. The purpose of the study was to determine EMG signal slops in submaximal trial of the trapezius muscle during shoulder shrugging.

This study has attempted to show the electromyographic activity of the upper trapezius muscle. Most reports on surface EMG spectral variables as indicators of localized muscle fatigue have focused on medium/high force contraction levels. However, low load contractions are more common at work place during long standing. It is known that the use of spectral variables to assess muscle fatigue under these conditions may be critical $[11,12]$. The objectives of this study are: 1 . To determine effect of fatigue on surface EMG parameters; 2. To comparison between healthy and patients fatigue effects.

There is no current evidence that muscle fatigue is effective in improving muscle flexibility and performance. The results of this original article suggest that fatigue may be used to increase flexibility, in spite of the very limited research. There is a need for high-quality clinical trials to evaluate the efficacy and effectiveness of fatigue and comparison between healthy and patients suffering upper trapezius myofascial trigger point.

\subsection{Materials and Methods}

This study was designed as a single-blind clinical trial and conducted at the clinic of Rehabilitation faculty, between April 2017 and December 2019.

\section{Subjects}

Thirty six right handed subjects without upper extremity disorders took part in this study. Healthy group (Mean \pm SD age: $29.78 \pm 6.28$ years, body mass index 22.50 \pm 1.74 , height $174.04 \pm 9.82 \mathrm{~cm}$, weight $68.44 \pm 9.7 \mathrm{~kg}$ ), and patient group (Mean \pm SD age, 22.70 \pm 4.48 years, Body Mass Index (BMI) 21.30 \pm 1.54 , height $170.04 \pm 9.71 \mathrm{~cm}$, weight $61.76 \pm 8.29 \mathrm{~kg}$ ). Matching of age, weight, height, and BMI was confirmed by the independent sample t-test.

\section{Inclusion criteria}

Individuals with an age range of 18-35 years were accepted for both groups to minimize any confounding effects stemming from advanced degenerative alterations in the cervical spine. The inclusion criteria for the patients group include: Restricted active or passive neck movement in at least one direction and score range of three to six points on an 11-points (range: zero to ten) Numerical Pain Rating Scale (NPRS) for perceived pain intensity. The inclusion criteria for the Control Group (CG) were no self-reported history of neck pain and no positive sign of cervical spine or scapular dysfunction during the physical examination.

\section{Exclusion criteria}

The following were the exclusion criteria for both groups:

History of neurological disorders (radiated pain) or neck surgery; chronic neck pain resulting from a traumatic incident; chronic musculoskeletal condition (such as muscular disorder or polyarthritis); medical diagnosis of fibromyalgia; systemic disease; connective tissue disorder; body mass index $<25 \mathrm{~kg} / \mathrm{m}$; non-tolerance of needles; currently pregnant; having undergone physical therapy, massage or acupuncture in the previous two weeks or use of analgesic, muscle relaxant, psychotropic agent or anti-inflammatory agent in the previous three days. 


\section{Outcome measures}

The outcome of the study was the surface EMG activity of the upper trapezius muscle after fatigue such as median frequency and RMS.

\section{Measures}

Surface recording electrodes were placed over the muscles as described by Cram et al [13]. while the ground electrode was placed on the ipsilateral wrist. To ensure good surface contact and to reduce skin resistance, a standard skin preparation procedure of disinfection, shaving and abrading was performed for each electrode placement site. Pre-gelled self-adhesive surface electrodes (Biometrics Ltd, electrode model SX230, 20_38mm) were then secured over the belly of the experimental muscles and aligned with their fibers' orientation on the both side of the participants [14].

\section{Experimental procedures}

The evaluations were performed with the patient seated comfortably in a chair with both feet flat on the digital balance, hips and knees flexed at $90^{\circ}$, buttocks positioned against the back of the chair and treated shoulder unclothed. Volunteers were asked to sit on the chair in an upright position with relaxed arms positioned by the sides of their body, as shown in Figure 1. The head was kept in the same position as the trunk and the vertebral column. The subjects were asked to look forward with no cervical and trunk rotation, extension or flexion during the test. Such was the position of the subjects.

\section{Fatigue protocol}

The protocol consisted of measuring the Maximal Voluntary Contraction (MVC) prior to the fatigue test, three times for approximately ten seconds, with a twenty seconds interval between tests. The highest value obtained was used to calculate the submaximal level at $(80 \%)$ of the MVC. The fatigue test was performed two minutes after the last MVC test.

The experimental protocol involved isometric contractions of the upper trapezius muscle on the subject's dominant side. During the experiment, the subjects were seated on a chair of adjustable height. Force sensors were placed on both shoulders so as to just touch the acromion when the subjects were sitting in a relaxed position with the arms hanging by the sides of the body. During sustained contractions of the trapezius, the subjects were asked to perform a shoulder elevation against the force transducers with the arms hanging passively by the sides of the body. Force was measured using a force
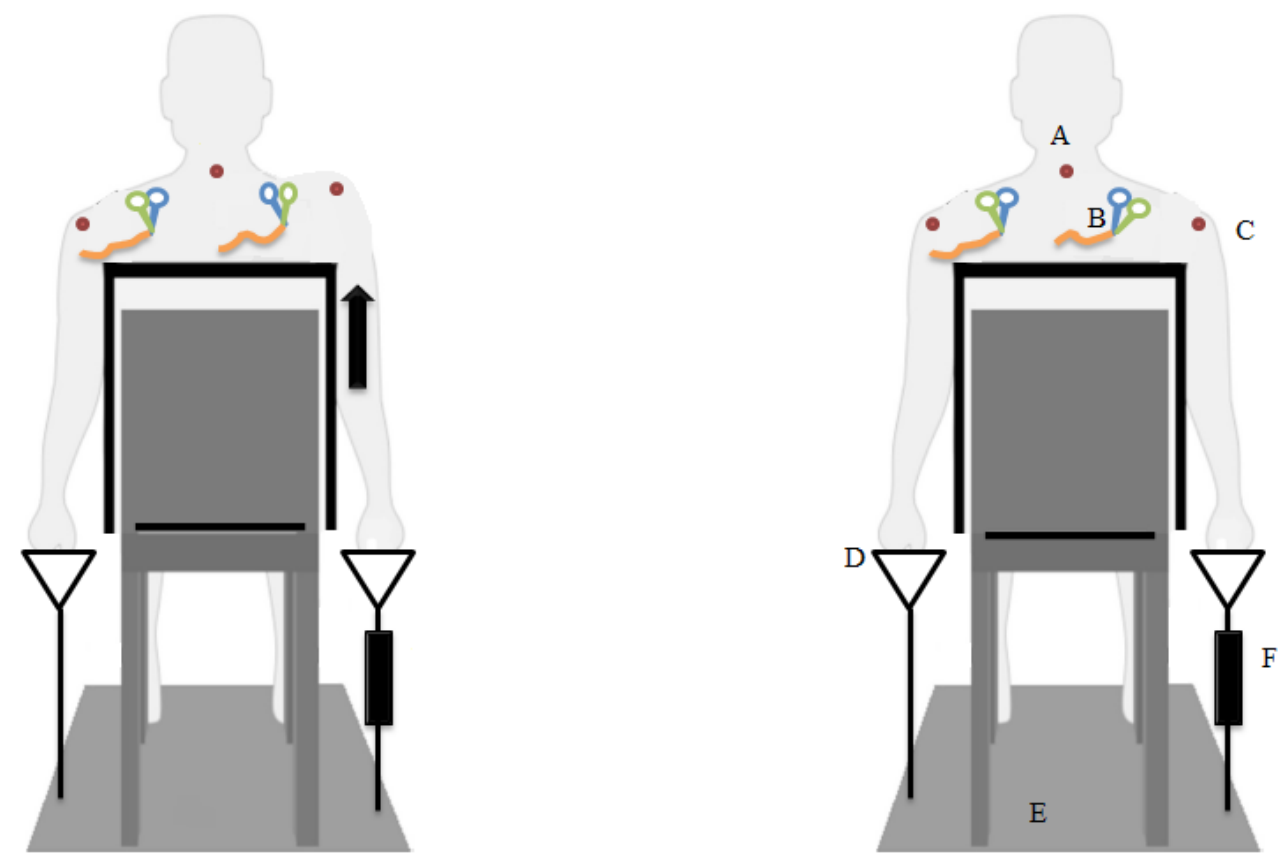

Figure 1. Details of experimental setup

1 Left image: Elevation of dominant shoulder against the load; 2 Right image: A. First position; B. Surface electrode; C. Acromion process; D. Handle of set up; E. Flat iron disc; F. Force gauge 
gauge (5020 model, Taiwan) and recorded on a PC (with sampling rate of $100 \mathrm{~Hz}$ ).

Measurement started with a Maximum Voluntary Contraction (MVC) force of the trapezius. In this case, the subjects were asked to perform a dominant shoulder elevation against the force transducer with as much force as possible for at least $10 \mathrm{~s}$. This procedure was repeated three times with in-between breaks of 20s. If the highest force was measured in the last MVC contraction, an additional contraction was performed.

Again the highest force value was considered to be the MVC. After a break of five min, a sustained submaximal contraction of the trapezius was performed. The subjects were asked to sustain a unilateral $(80 \%)$ MVC isometric shoulder elevation until the force gauge monitor showed $(50 \%)$ of MVC in at least three min. The subjects received strong verbal encouragement during the MVC and the fatigue test. Although no subjective assessment of fatigue was done, the subjects showed exhaustion at the end of the protocol. The force level corresponding to $(80 \%)$ of the MVC was chosen because the protocol aimed to trigger muscle fatigue.

\section{Statistical analysis}

The Kolmogorov-Smirnov test was used to assess the normal distribution of the data, for both groups (MTP group: 18; non-MTP group: 18), Intra-class Correlation Coefficients (ICCs) with (95\%) Confidence Intervals (CIs) were used to determine relative reliability between two trials. Standard Errors of the Measurements (SEMs) were used to determine measurement precision in the same unit. Standard deviation and ICCs were used to calculate the SEM for each measure. ICC values were considered very high if $>0.90$, high if between 0.70 0.89 , and moderate if between 0.50-0.69 [15]. SEM was deemed acceptable if $<10 \%$ [16].

For assessing the difference in strength between the non-MTP and MTP groups, independent t-tests were used to compare the strength of the scapular elevator, shoulder abductor, and shoulder abductor with restricted scapular elevation between the groups. The level of statistical significance was set at 0.05 . The SPSS V. 25 software was used for all analyses.

\section{Results}

To evaluate the fatigue protocol, Median Frequency (MF) and Root Mean Square (RMS) of the upper trapezius muscle were evaluated. The MF and RMS param- eters of the left and right sides of both healthy subjects and patients were significantly different. The RMS norm of the sustained trapezius contractions showed differences between the groups. Changes of median frequency and RMS were higher in the healthy subjects than the patients group and these changes in the right side were higher than in the left side (Table $1 \& 2$ ).

\section{Discussion}

Under controlled conditions, the EMG signal is often used as an indicator of muscle fatigue and the corresponding physiological changes [17, 18]. The Fast Fourier Transform (FFT) and subsequent mean and median frequency calculations often demonstrate a spectrum shift to lower frequencies during sustained muscle contractions $[7,17,18]$.

Some authors have suggested that during sustained isometric contraction with $(50 \%)$ or greater, of maximal force, the accumulation of lactic acid leads to decreased $\mathrm{pH}$ in contracting muscles $[14,16]$. This accumulation also inhibits the excitability of the muscle membrane, thereby causing a decrease in the firing rate and, consequently, a decrease in MF. The shift in median frequency provides confirmatory evidence that the decline in force production was due to fatigue and not due to lack of effort [19-21].

RMS is a technique used to describe changes in EMG amplitude and was shown to be somewhat sensitive to fatigue-related changes in this study. Even though it allows for the quantification of muscle activation, RMS provides a single value to represent the entire contraction of interest. The near-infinite number of activation profiles during a movement has been previously described as the muscle redundancy problem [19]. Therefore, the validity of RMS becomes questionable due to possible changes in activation strategies occurring at different time-points throughout the dynamic task and thus, the observed differences cannot be limited to those caused by fatigue [22].

There were no statistical differences in the fatigability properties of the two sides. This is likely related to differences in the subcutaneous layer thickness in the two groups, which is gender and age dependent [23]. Such divergences may arise from differences in characteristics such as the subject's resistance to fatigue, the contraction properties of the skeletal muscle, muscle fiber composition, enzyme activity levels, differences in metabolic systems and differences in signal processing, between individual subjects. Despite the characteristics of indi- 
Table 1. Mean \pm SD for the RMS (mV) for both groups

\begin{tabular}{cccccccc}
\hline \multicolumn{1}{c}{ Variables } & Mean & Std. Deviation & Std. Error Mean & Lower & Upper & T & Sig \\
\hline RMS- healthy- right & -11.65846 & 8.31181 & 2.30528 & -16.68124 & -6.63569 & -5.057 & 0.000 \\
\hline RMS- healthy- left & -10.59462 & 4.92563 & 1.36612 & -13.57114 & -7.61809 & -7.755 & 0.000 \\
RMS- patient- right & -11.96200 & 4.91703 & 1.55490 & -15.47943 & -8.44457 & -7.693 & 0.000 \\
RMS- patient- left & -10.81000 & 2.45392 & 0.77600 & -12.56543 & -9.05457 & -13.930 & 0.000 \\
\hline
\end{tabular}

Table 2. Healthy and neck pain groups variable before \& after fatigue

\begin{tabular}{ccccccc}
\hline Variables & Mean \pm SD & Std. Error Mean & Lower & Upper & Tig \\
\hline MDF- healthy- left & $5.11500 \pm 22.74245$ & 5.68561 & -7.00360 & 17.23360 & 0.900 & 0.383 \\
MDF- healthy- right & $17.2881 \pm 8.87433$ & 2.21858 & 12.55933 & 22.01692 & 7.792 & 0.000 \\
MDF- patient- right & $9.05050 \pm 7.26436$ & 1.62436 & 5.65067 & 12.45033 & 5.572 & 0.000 \\
MDF- patient- right & $32.0490 \pm 13.48586$ & 3.01553 & 25.73742 & 38.36058 & 10.628 & 0.000 \\
\hline
\end{tabular}

vidual subjects, divergent RMS behavior was consistent with the muscle group in the present study. The RMS increased in the right upper trapezius, for groups with and without pain.

In the present study, this behavior was noted in the upper trapezius muscle. In contrast, some studies have indicated that the RMS value behaves differently. The occlusion of blood flow due to intramuscular pressure during static contraction leads to a reduction in the oxygen supply to the active muscle [14]. Consequently, according to Vas MA, glycolytic metabolism increases, and there is an accumulation of lactate [24]. In order to compensate for this situation and maintain force levels, recruitment of new Motor Units (MUs) can be expected. This motor unit recruitment is reflected in the EMG signal amplitude and, therefore, can be identified by calculating the Root Mean Square (RMS) [25-27].

The increase in RMS is related to the recruitment of additional motor units and also an increased firing rate. These are necessary to compensate for the loss of force [28]. The spectral compression produced by the slower conduction of action potential also increases the RMS. In the present study, this behavior was noted in the upper trapezius muscle [29]. In contrast, some studies have indicated that the RMS value behaves differently. For example, Gerdle et al. 2009 reported that the RMS value showed various patterns within the same group (increased, unchanged or decreased) during the fatigue phase [30]. Despite the characteristics of individual subjects, divergent RMS behavior was consistent with the muscle group in the present study. The RMS increased in the right upper trapezius.

\section{Conclusion}

There was a significant increase in RMS amplitude from the beginning to the end of the fatigue protocol for each group. The fact that our patient group showed similar decreases in MDF (and an increase in RMS) as the controls after a much shorter time (fewer contractions), provides further support that the patient group may have fewer type I fibers.

The change in MDF of patient was greater than healthy controls, which suggests the prevalence of type II fibers or greater fatigability and reduced endurance of the superficial cervical flexors in myofascial trigger point patients. The fact that our NP group showed similar decreases in MDF (and an increase in RMS) as the controls after a much shorter time (fewer contractions), provides further support that the patient group may have fewer type I fibers. There was a significant decrease in MDF from the beginning to the end of the fatigue protocol for both the controls and patient groups. 
The effects of other muscles attached to scapula or related to shoulder elevation and any deficits in these muscles on movement quality were not assessed. As mentioned before, the upper trapezius muscle was chosen, because of its key role in dynamic stability of scapular during upper limb elevation and the strong possibility for the presence of LMTrPs in UT muscle. Another limitation of this study was that the amplitude of the muscle activations during movement and muscles related to shoulder elevation were not taken into account. Moreover, all participants in this study were females with a very narrow age range and the findings cannot be extrapolated to all female age groups.

\section{Ethical Considerations}

\section{Compliance with ethical guidelines}

All the procedures were carried out after the study was approved by the Scientific Board and Ethics Committee of the School of Rehabilitation, Tehran University of Medical Science (IRCT2017011426346N2). Ethics Committee number IR.TUMS.VCR.REC 1395.803; 2016/10/22. All participants were informed about the procedures that would be carried out. The participants who agreed voluntarily to sign an identified consent form were considered for the study.

\section{Funding}

The present paper was extracted from the MSc. Thesis of the first author, in International Campus, School of Rehabilitation, Tehran University of Medical Sciences.

\section{Authors contributions}

Carrying out the literature search and review: Roya Mehdikhani, Gholam Reza Olyaei, Mohammad Reza Hadian and Saeed Talebian Moghaddam; data extracting, synthesizing results, preparing the initial draft, performing the statistical analysis, submitting the manuscript: Roya Mehdikhani; preparing the research framework, coordinating appraisal, data critical appraisal, preparing the manuscript revisions: Gholam Reza Olyaei, Mohammad Reza Hadian and Saeed Talebian Moghaddam; Search strategy and revisions of the manuscript, Statistical suggestions and analysis: Saeed Talebian Moghaddam; Reading and approving the final article: All authors.

\section{Conflict of interest}

The authors declared no conflict of interests.

\section{Acknowledgements}

The authors thank Mr. Mohammad Taghi Moghaddam Fard, head of the board director of Iran Exhaust Company, for his help in designing the experiment.

\section{References}

[1] Cimbiz A, Beydemir F, Manisaligil U. Evaluation of trigger points in young subjects. Journal of Musculoskeletal Pain. 2006; 14(4):27-35. [DOI:10.1300/J094v14n04_04]

[2] Lucas KR, Rich PA, Polus BI. How common are latent myofascial trigger points in the scapular positioning muscles? Journal of Musculoskeletal Pain. 2008; 16(4):279-86. [DOI:10.1080/10582450802479800]

[3] Simons DG, Travell JG, Simons LS. Myofascial pain and dysfunction: the trigger point manual. Upper Half of Body. 1999; 17(1):72.

[4] Johnson G, Bogduk N, Nowitzke A, House D. Anatomy and actions of the trapezius muscle. Clinical Biomechanics. 1994 9(1):44-50. [DOI:10.1016/0268-0033(94)90057-4]

[5] Barry DT, Geiringer SR, Ball RD. Acoustic myography: A noninvasive monitor of motor unit fatigue. Muscle \& Nerve. 1985; 8(3):189-94. [DOI:10.1002/mus.880080303] [PMID]

[6] Bigland-Ritchie B, Furbush F, Woods JJ. Fatigue of intermittent submaximal voluntary contractions: Central and peripheral factors. Journal of Applied Physiology. 1986; 61(2):421-9. [DOI:10.1152/jappl.1986.61.2.421] [PMID]

[7] De CL. Myoelectrical manifestations of localized muscular fatigue in humans. Critical Reviews in Biomedical Engineering. 1984; 11(4):251-79. [PMID]

[8] Orizio C. Muscle sound: Bases for the. Journal of Critical Reviews in Biomedical Engineering. 1993;21(3):201-43.

[9] Orizio C, Perini R, Diemont B, Veicsteinas A. Muscle sound and electromyogram spectrum analysis during exhausting contractions in man. European Journal of Applied Physiology and Occupational Physiology. 1992; 65(1):1-7. [DOI:10.1007/ BF01466266

[10] Schüldt K, Harms-Ringdahl K. Activity levels during isometric test contractions of neck and shoulder muscles. Scandinavian Journal of Rehabilitation Medicine. 1988; 20(3):117-27. [DOI:10.1016/0268-0033(88)90046-0]

[11] Farina D, Gazzoni M, Merletti R. Assessment of low back muscle fatigue by surface EMG signal analysis: Methodological aspects. Journal of Electromyography and Kinesiology. 2003; 13(4):319-32. [DOI:10.1016/S1050-6411(03)00040-3]

[12] Öberg TO, Sandsjö LE, Kadefors R. Subjective and objective evaluation of shoulder muscle fatigue. Ergonomics. 1994 37(8):1323-33. [DOI:10.1080/00140139408964911] [PMID]

[13] Cram JR, Kasman G, Holtz J. Introduction to surface electromyography aspen publishers. Gaithersburg: Aspen Publisher; 1998. 
[14] Masuda K, Masuda T, Sadoyama T, Inaki M, Katsuta $\mathrm{S}$. Changes in surface EMG parameters during static and dynamic fatiguing contractions. Journal of Electromyography and Kinesiology. 1999; 9(1):39-46. [DOI:10.1016/S10506411(98)00021-2]

[15] Seburn KL, Sanderson DJ, Belcastro AN, McKenzie DC. Effect of manipulation of plasma lactate on integrated EMG during cycling. Medicine and Science in Sports and Exercise. 1992; 24(8):911-6. [DOI:10.1249/00005768-199208000-00013]

[16] Tsuboi T, Satou T, Egawa K, Izumi Y, Miyazaki M. Spectral analysis of electromyogram in lumbar muscles: Fatigue induced endurance contraction. European Journal of Applied Physiology and Occupational Physiology. 1994; 69(4):361-6. [DOI:10.1007/BF00392044]

[17] De Luca CJ. Use of the surface EMG signal for performance evaluation of back muscles. Muscle \& Nerve. 1993; 16(2):2106. [DOI:10.1002/mus.880160216] [PMID]

[18] Merletti R, Rainoldi A, Farina D. Surface electromyography for noninvasive characterization of muscle. Exercise and Sport Sciences Reviews. 2001; 29(1):20-5. [DOI:10.1097/00003677200101000-00005] [PMID]

[19] Buchanan TS, Shreeve DA. An evaluation of optimization techniques for the prediction of muscle activation patterns during isometric tasks. Journal of Biomechanical Engineering. 1996; 118(4):565-74. [DOI:10.1115/1.2796044] [PMID]

[20] Merletti R, Knaflitz M, De Luca CJ. Myoelectric manifestations of fatigue in voluntary and electrically elicited contractions. Journal of Applied Physiology. 1990; 69(5):1810-20. [DOI:10.1152/jappl.1990.69.5.1810] [PMID]

[21] Westgaard RH, Winkel J. Ergonomic intervention research for improved musculoskeletal health: A critical review. International Journal of Industrial Ergonomics. 1997; 20(6):463-500 [DOI:10.1016/S0169-8141(96)00076-5]

[22] Treleaven J, Jull G, LowChoy N. The relationship of cervical joint position error to balance and eye movement disturbances in persistent whiplash. Manual Therapy. 2006;11(2):99-106. [DOI:10.1016/j.math.2005.04.003] [PMID]

[23] Zabihhosseinian M, Holmes MW, Ferguson B, Murphy B. Neck muscle fatigue alters the cervical flexion relaxation ratio in sub-clinical neck pain patients. Clinical Biomechanics. 2015; 30(5):397-404. [DOI:10.1016/j.clinbiomech.2015.03.020] [PMID]

[24] Vaz MA, Zhang YT, Herzog W, Guimaraes AC, MacIntosh $\mathrm{BR}$. The behavior of rectus femoris and vastus lateralis during fatigue and recovery: An electromyographic and vibromyographic study. Electromyography and Clinical Neurophysiology. 1996; 36(4):221-30. [PMID]

[25] Jensen C, Westgaard RH. Functional subdivision of the upper trapezius muscle during maximal isometric contractions. Journal of Electromyography and Kinesiology. 1995; 5(4):22737. [DOI:10.1016/1050-6411(94)00011-5]

[26] Mathiassen SE, Winkel J, Hägg GM. Normalization of surface EMG amplitude from the upper trapezius muscle in ergonomic studies-a review. Journal of Electromyography and Kinesiology. 1995; 5(4):197-226. [DOI:10.1016/10506411(94)00014-X]
[27] Roy SH, De CL, Casavant DA. Lumbar muscle fatigue and chronic lower back pain. Spine. 1989; 14(9):992-1001. [DOI:10.1097/00007632-198909000-00014] [PMID]

[28] Kleine BU, Schumann NP, Stegeman DF, Scholle HC. Surface EMG mapping of the human trapezius muscle: The topography of monopolar and bipolar surface EMG amplitude and spectrum parameters at varied forces and in fatigue. Clinical Neurophysiology. 2000; 111(4):686-93. [DOI:10.1016/ S1388-2457(99)00306-5]

[29] Mork PJ, Westgaard RH. Long-term electromyographic activity in upper trapezius and low back muscles of women with moderate physical activity. Journal of Applied Physiology. 2005; 99(2):570-8. [DOI:10.1152/japplphysiol.00198.2005] [PMID]

[30] Gerdle B, Larsson B, Karlsson S. Criterion validation of surface EMG variables as fatigue indicators using peak torque: A study of repetitive maximum isokinetic knee extensions. Journal of Electromyography and Kinesiology. 2000; 10(4):225-32 [DOI:10.1016/S1050-6411(00)00011-0] 
This Page Intentionally Left Blank 